\title{
Omental gastrointestinal stromal tumor: Arriving at a diagnosis with therapeutic options in the molecular era
}

\author{
Michael P. Greenwood ${ }^{1}$, F. Jariel Hairston ${ }^{2}$, Mary R Schwartz ${ }^{1}$, Kristi Pepper ${ }^{1}$, Heather Hendrickson ${ }^{1}$, Anuj Suri ${ }^{3}$, \\ Donna Coffey ${ }^{1}$, Michael Deavers ${ }^{1}$, Randall J. Olsen ${ }^{1}$, Jessica S. Thomas*1 \\ ${ }^{1}$ Pathology \& Genomic Medicine, Houston Methodist, Houston, USA \\ ${ }^{2}$ North Mississippi Medical Center, Tupelo, USA \\ ${ }^{3}$ Houston Methodist Gynecologic Oncology Associates, Houston Methodist, Houston, USA
}

Received: April 19, 2017

Accepted: June 22, 2017

Online Published: July 3, 2017

DOI: $10.5430 /$ crcp.v4n3p7

URL: https://doi.org/10.5430/crcp.v4n3p7

\begin{abstract}
Omental extragastrointestinal stromal tumors are rare neoplasms. We present a case of an elderly woman who was found to have a large solid and cystic pelvic mass of presumed ovarian origin with associated omental neovascularity. Histopathologic examination showed a mixed spindled and epithelioid tumor which was essentially negative for c-KIT and positive for smooth muscle markers by immunohistochemistry. A next generation sequencing panel was performed, analyzing the patient's tumor for actionable mutations in 50 genes commonly associated with human cancers. Identification of a point mutation in PDGFRA enabled accurate diagnosis and provided an option for targeted therapy for this rare tumor.
\end{abstract}

Key Words: Gastrointestinal stromal tumor, PDGFRA mutation, Molecular diagnostics, Targeted therapy

\section{INTRODUCTION}

Gastrointestinal stromal tumors (GISTs), derived from the interstitial cells of Cajal, are the most common mesenchymal neoplasm involving the gastrointestinal system. They account for less than $1 \%$ of all gastrointestinal tumors and approximately $5 \%$ of all sarcomas. ${ }^{[1]}$ They may occur at any site within the tubular gastrointestinal tract, with the most common sites of involvement being stomach (60\%-70\%) and small bowel (20\%-30\%). ${ }^{[2]}$ Sporadic GISTs are most common, though familial GISTs have been well-described and may present as part of several inherited tumor syndromes. ${ }^{[1]}$ The molecular etiology of GISTs most commonly involves ligand independent activation of the c-KIT signaling pathway through mutation of the KIT gene (80\%) and less commonly $(5 \%-10 \%)$ involves a gain of function mutation in the
PDGFRA gene. ${ }^{[1,2]}$ Approximately 9\%-15\% of all GISTs are termed "wild-type" and show no evidence of mutations in either KIT or PDGFRA. ${ }^{[1]}$ Prior to molecular characterization of these tumors, GISTs were often miscategorized as leiomyosarcomas due to significant morphologic overlap and occasional presentation in atypical anatomic sites. Extragastrointestinal intestinal stromal tumors (EGISTs) are a minor subset of GISTs which may occur in the omentum, mesentery, pelvic cavity, and retroperitoneum, together reported to represent from $0-8 \%$ of all GISTs. ${ }^{[3]}$ Due to their overall rarity, the clinicopathologic features of EGISTs are not well recognized. We present a case in which molecular characterization allowed for accurate diagnosis of and provided treatment options for an omental EGIST harboring a PDGFRA mutation.

\footnotetext{
*Correspondence: Jessica S. Thomas, MD, PhD, MPH; Email: jsthomas@ houstonmethodist.org; Address: 6565 FANNIN ST, MS205, Department of Pathology \& Genomic Medicine, Houston Methodist Hospital, Houston, TX, 77030, USA.
} 


\section{CASE PResentation}

A 79-year-old female presented with back pain. Her history was significant for Alzheimer disease, hypertension, left hip replacement, and prior total abdominal hysterectomy with unknown pathology. The patient's serum CA-125 was found to be elevated at $195 \mathrm{U} / \mathrm{ml}$ and her serum CEA was within normal limits. Computed tomography of the abdomen and pelvis showed a large solid and cystic mass in the right pelvis, favored to be of ovarian origin, accompanied by neovascularity in the omentum. The patient was taken for exploratory laparotomy and a $20 \mathrm{~cm}$, relatively well-circumscribed soft hemorrhagic omental mass was resected (see Figure 1). No other sites of tumor were identified in the pelvis or abdomen.

Histopathologic assessment of the mass demonstrated a cellular neoplasm with mixed areas of spindled and epithelioid morphology, as shown in Figure 2. No significant nuclear pleomorphism or increased mitotic activity was identified, although there was focal infarct-type tumor necrosis. Immunohistochemical studies (see Figure 3) demonstrated strong diffuse positivity for three smooth muscle markers: desmin, caldesmon, and smooth muscle actin. Although there was strong diffuse immunoreactivity for DOG-1, there was only focal weak immunostaining for c-kit (CD117) which was interpreted as negative. Immunohistochemical staining for pancytokeratin, S-100 protein, and inhibin was negative. While DOG-1 is generally considered a sensitive marker for GIST, it is not $100 \%$ specific and, in one study, was expressed in seven of 26 (27\%) uterine leiomyosarcomas. ${ }^{[5]}$ Based on these findings, along with the patient's clinical history and the lack of a gastrointestinal association, an initial diagnosis of leiomyosarcoma was favored.

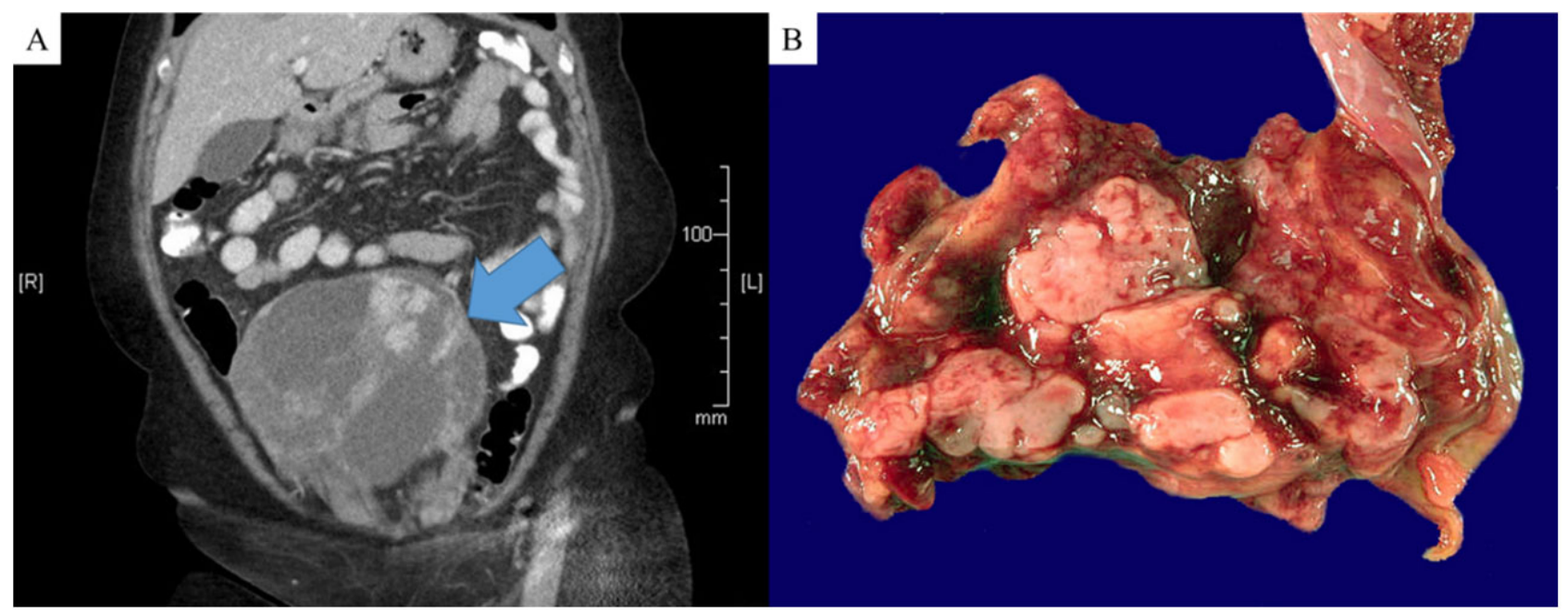

Figure 1. A) Coronal computed tomography and B) gross images of the tumor

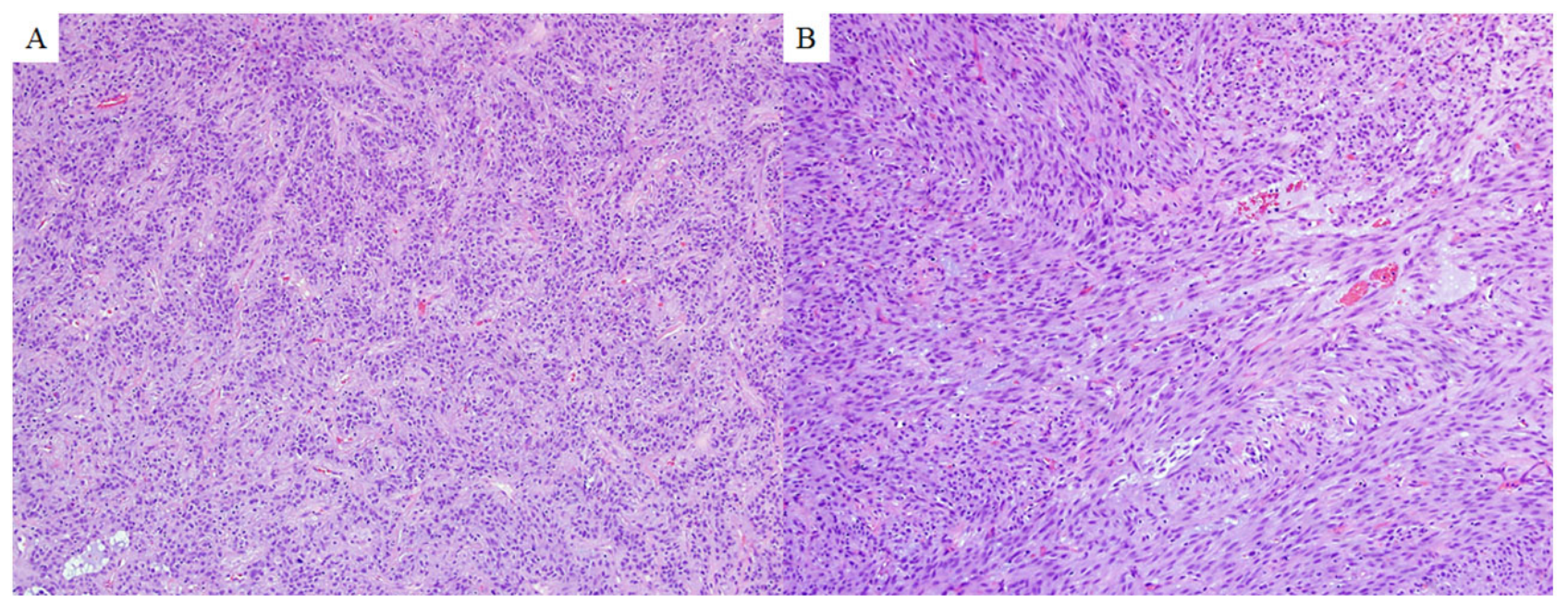

Figure 2. Hematoxylin and eosin stained sections of the patient's tumor, showing the distinct A) epithelioid and B) spindled areas of the tumor $(100 \times$ original magnification $)$ 


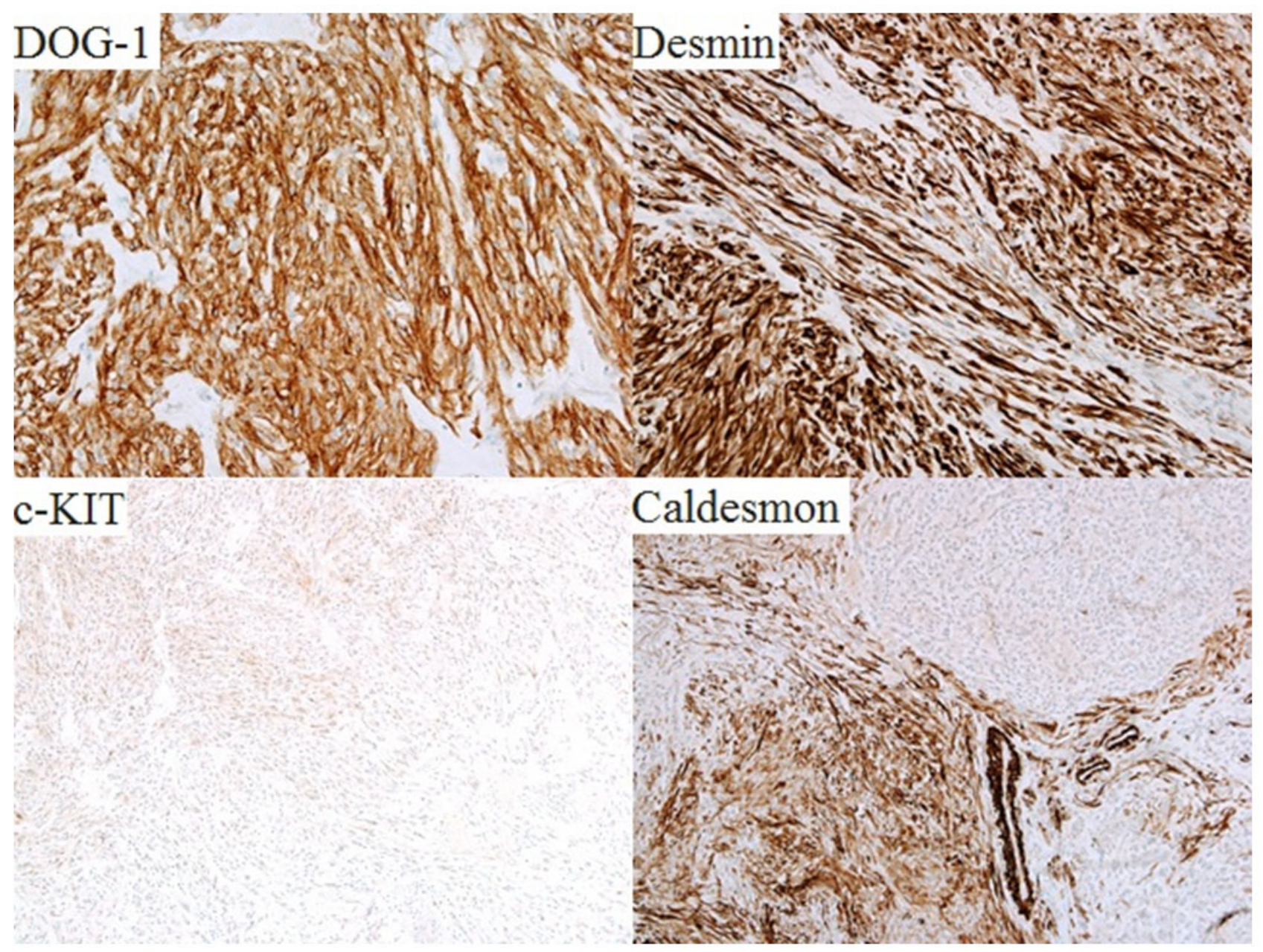

Figure 3. Immunohistochemically stained sections of the patient's tumor, showing strong positivity for DOG-1, desmin, and caldesmon, and focal very weak positivity for CD117 (c-KIT)

Molecular analysis of the tumor was performed on formalinfixed paraffin-embedded tissue. A panel of 50 genes commonly mutated in human cancers was interrogated by next generation sequencing (Ion AmpliSeq Cancer Hotspot Test v2) using the Ion Torrent PGM, including: $A B L 1, A K T 1$, $A L K, A P C, A T M, B R A F, C D H 1, C D K N 2 A, C S F 1 R, C T N N B 1$, EGFR, ERBB2, ERBB4, EZF2, FBXW7, FGFR1, FGFR2, FGFR3, FLT3, GNA11, GNAQ, GNAS, HNF1A, HRAS, IDH1, IDH2, JAK2, JAK3, KDR, KIT, KRAS, MET, MLH1, MPL, NOTCH1, NPM1, NRAS, PDGFRA, PIK3CA, PTEN, PTPN11, RB1, RET, SMAD4, SMARCB1, SMO, SRC, STK11, $T P 53$, and $V H L$. The lower limit of detection of this assay (analytical sensitivity) is 10\% at 100x-fold coverage, and no amplicons were excluded from analysis due to low coverage. Four variants were identified in the molecular analysis of this patient's tumor using Houston Methodist Variant Viewer (an in-house developed and clinically validated bioinformatics pipeline), as shown in Table 1. Of these, only the
PDGFRA c.1682T >A (p.Val561Asp) variant was identified as a known somatic mutation in cancer. As shown in Figure 4 , sequencing of the patient's tumor shows the point mutation c. $1682 \mathrm{~T}>\mathrm{A}$ in $36 \%$ of PDGFRA gene reads. The other three variants identified were found in TP53, STK11, and GNA11, respectively (see Table 1). Each of these variants was further investigated, as per laboratory protocol, using our reporting criteria and the Integrated Genomics Viewer ${ }^{[4]}$ analysis of the sequencing data. The TP53 variant was present at a very low frequency and the STK11 variant was only seen in sequencing reads in the reverse direction, suggesting that these are both amplification or sequencing errors. The GNA11 variant is a deletion of a guanine in a homopolymer of six guanine residues, a platform specific sequencing error seen with the Ion Torrent instrument. Of the four variants initially identified, only the PDGFRA variant was verified as a reportable somatic mutation detected in this patient's tumor. 
Table 1. All variants identified by 50 gene next generation sequencing panel

\begin{tabular}{llllll}
\hline Variant & Consequence & $\begin{array}{l}\text { Variant allele } \\
\text { fraction }\end{array}$ & $\begin{array}{l}\text { Depth of } \\
\text { coverage }\end{array}$ & dbSNP & COSMIC \\
\hline $\begin{array}{l}\text { ENST257290.5 (PDGFRA): c.1682T>A } \\
\text { (p.Val561Asp) }\end{array}$ & $\begin{array}{l}\text { Substitution - } \\
\text { Missense }\end{array}$ & 0.35807 & 1994 & rs121908586 & COSM739 \\
$\begin{array}{l}\text { ENST269305.4 (TP53): c.270dupC } \\
\text { (p.Tr91LeufsTer58) }\end{array}$ & $\begin{array}{l}\text { Duplication }- \\
\text { frameshift }\end{array}$ & 0.01832 & 1436 & & \\
$\begin{array}{l}\text { ENST326873.7 (STK11): c.173delG } \\
\text { (p.Gly58AlafsTer6) }\end{array}$ & $\begin{array}{l}\text { Deletion - } \\
\text { frameshift }\end{array}$ & 0.40681 & 1979 & & \\
$\begin{array}{l}\text { ENST78429.4 (GNA11): c.623delG } \\
\text { (p.Gly208AlafsTer16) }\end{array}$ & $\begin{array}{l}\text { Deletion - } \\
\text { frameshift }\end{array}$ & 0.45443 & 1538 & & COSM1392334 \\
\hline
\end{tabular}

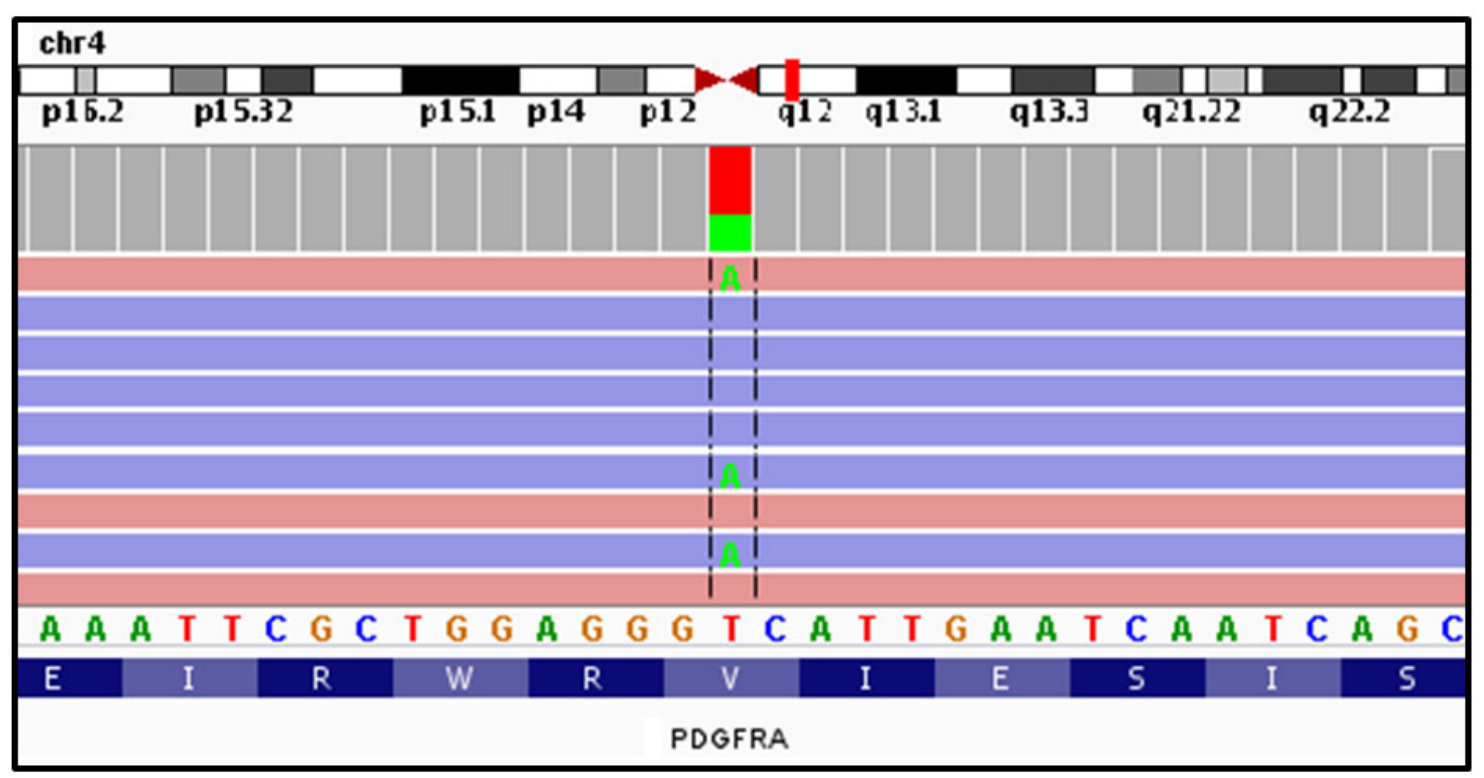

Figure 4. Integrated Genomics Viewer ${ }^{[4]}$ representative display of the mutated sequence in PDGFRA. Nine separate sequencing reads at this nucleotide position (1682) are shown, 3 of which demonstrate the T>A substitution (the variant allele frequency for this mutation was 36\%). This point mutation causes an amino acid substitution (Aspartic acid for Valine) at codon 561 in exon 12 of PDGFRA.

\section{Discussion}

In this case of an EGIST presenting as a solid and cystic omental mass, sequencing of the tumor for somatic variants using a 50 gene cancer panel helped to clarify ambiguous morphologic and immunohistochemical findings. The tumor had an uncommon presentation coupled with a mixed spindled and epithelioid morphology and an overall immunohistochemical profile most consistent with a diagnosis of leiomyosarcoma. However, the presence of the PDGFRA exon 12 mutation by molecular analysis supported the diagnosis of a GIST in this case. ${ }^{[4]}$

Although GISTs may infrequently occur outside of the GI tract, these EGISTs are usually immunoreactive for CD117 (c-KIT) and negative for desmin by immunohistochemistry. ${ }^{[2]}$ Of note, desmin immunoreactivity is reported to be more common in omental GISTs than in gastric or small intestinal GISTs. ${ }^{[3]}$ CD117-negative EGISTs are uncommon involving only 3 of 41 solitary omental GISTs in one study. ${ }^{[3]}$ An additional study of the clinicopathologic and genetic features of 10 CD117-negative solitary EGISTs suggested that these tumors may be characterized by preferential omental origin, epithelioid cell type, low mitotic activity, and mutation of the PDGFRA gene. ${ }^{[6]}$ Immunoreactivity for DOG-1 by immunohistochemistry has been reported in $90 \%$ of EGISTs. ${ }^{[5,6]}$

Of these genes analyzed in this case, mutually exclusive mutations in KIT, PDGFRA, and BRAF have been reported to occur in GISTs. ${ }^{[7]}$ The exon 12 PDGFRA mutation identified in this case is a recurrent activating mutation found in approximately $14 \%$ of PDGFRA-mutated GISTs. ${ }^{[8,9]}$ This mutation has previously been reported in 1 of a series of 10 cases of CD117-negative omental EGISTs ${ }^{[6]}$ and in 1 in a series of 8 
PDGFRA-mutated solitary omental EGISTs. ${ }^{[3]}$ Unlike the exon 18 PDGFRA D842V mutation, the less frequent exon 12 PDGFRA V561D mutation is known to confer increased sensitivity to imatinib. ${ }^{[9,10]}$ Based on the mutational profile of this patient's tumor, personalized targeted therapy with imatinib was recommended. Unfortunately, the patient relocated prior to the initiation of imatinib therapy and follow up on her outcome was not available.

Analysis of tumor DNA for the presence of somatic mutations are now sought to guide diagnosis, assist with deter- mination of prognosis, and guide personalized therapeutic strategies for patients. In this case, the clinical features and an unusual immunohistochemical staining pattern initially favored a more likely diagnosis. However, somatic mutational analysis of this patient's tumor aided in obtaining the correct diagnosis, as well as providing an option for highly effective targeted therapy.

\section{CONFLICTS OF INTEREST Disclosure}

the authors declare no conflicts of interest.

\section{REFERENCES}

[1] Zhao X, Yue C. Gastrointestinal Stromal Tumor. J Gastrointest Oncol. 2012 Sep; 3(3): 189-208. PMid: 22943011.

[2] Miettinen M, Lasota J. Gastrointestinal stromal tumors: pathology and prognosis at different sites. Semin Diagn Pathol. 2006 May; 23(2): 70-83. PMid: 17193820. https://doi.org/10.1053/j. semdp.2006.09.001

[3] Miettinen M, Sobin LH, Lasota J. Gastrointestinal stromal tumors presenting as omental masses-a clinicopathologic analysis of 95 cases. Am J Surg Pathol. 2009 Sep; 33(9): 1267-75. https : //doi. org/10.1097/PAS.0b013e3181a13e99

[4] Thorvaldsdóttir H, Robinson JT, Mesirov JP. Integrative Genomics Viewer (IGV): high-performance genomics data visualization and exploration. Brief Bioinform. 2013 Mar; 14(2): 178-192. PMid: 22517427. https://doi.org/10.1093/bib/bbs017

[5] Sah SP, McCluggage WG. DOG1 immunoreactivity in uterine leiomyosarcomas. J Clin Pathol. 2012 Dec 23; 66(1): 40-3. https : //doi.org/10.1136/jclinpath-2012-201150

[6] Yamamoto H, Kojima A, Nagata S, et al. KIT-Negative Gastrointestinal Stromal Tumor of the Abdominal Soft Tissue: A Clinicopatho- logic and Genetic Study of 10 Cases. Am J Surg Pathol. 2011 Sep; 35(9): 1287-1295. PMid: 21836495. https://doi.org/10.109 7/PAS. Ob013e3182206f 15

[7] Rubin BP, Heinrich MC. Genotyping and immunohistochemistry of gastrointestinal stromal tumors: An update. Semin Diagn Pathol. 2015; 32(5): 392-9. PMid: 25766843. https ://doi.org/10.105 3/j.semdp. 2015.02.017

[8] Hirota S, Ohashi A, Nishida T, et al. Gain-of-function mutations of platelet-derived growth factor receptor alpha gene in gastrointestinal stromal tumors. Gastroenterology. 2003 Sep; 125(3): 660-7. https://doi .org/10.1016/S0016-5085(03)01046-1

[9] Corless CL, Schroeder A, Griffith D, et al. PDGFRA mutations in gastrointestinal stromal tumors: frequency, spectrum and in vitro sensitivity to imatinib. J Clin Oncol. American Society of Clinical Oncology; 2005 Aug 10; 23(23): 5357-64. https ://doi.org/10 $.1200 /$ JC0. 2005.14 .068

[10] Heinrich MC, Corless CL, Demetri GD, et al. Kinase mutations and imatinib response in patients with metastatic gastrointestinal stromal tumor. J Clin Oncol. 2003 Dec 1; 21(23): 4342-9. http: //dx.doi.org/10.1200/JC0.2003.04.19 